Artigo de Revisão

\title{
Fisiologia e importância do órgão tendinoso de Golgi no controle motor normal
}

\section{Physiology and importance of Golgi tendon organ for the normal motor control}

Marco Taneda'1, José Eduardo Pompeu²

\section{RESUMO}

Introdução: Os órgãos tendinosos de Golgi (OTG) são receptores sensoriais localizados na junção miotendínea e conectados em série com as fibras musculares. Sua função no controle motor em humanos ainda não está elucidada. Objetivos: Relatar a fisiologia do OTG e seu envolvimento em distúrbios do tônus e na percepção da força, bem como sua interação com outros sistemas envolvidos no controle motor. Métodos: A revisão da literatura foi realizada no período de 1964 até os dias atuais. O estudo foi baseado em artigos experimentais e de revisão além de capítulos de livros-textos. Resultados: O OTG é inervado por axônio aferente (af) lb que dispara sempre que há tensão no músculo, inibindo, através de um interneurônio (IN) inibitóriolb, o neurônio motor que está gerando a força. Além disso, os neurônios motores antagonistas são facilitados através de duas sinapses por um IN excitatório lb. Os estímulos convergem ao IN lb das mais variadas fontes, incluindo vários af periféricos e estímulos excitatórios e inibitórios suprassegmentares. Conclusão: O OTG atua juntamente com o fuso muscular na regulação do tônus e da complacência musculares; está envolvido na mediação da propriocepção e possui um papel importante no ritmo locomotor normal.

\section{Unitermos: Mecanorreceptores, Propriocepção, Tono muscular.}

Citação: Taneda M, Pompeu JE. Fisiologia e importância do órgão tendinoso de Golgi no controle motor normal. Rev Neurocienc 2006; 14(1):037-042.

\section{SUMMARY}

Introduction: The Golgi tendon organs (GTO) are sensorial receptors situated between the muscle and his tendon and they are connected in series with the muscle fibers. Your function on human motor control is still not elucidated. Objectives: To present the GTO physiology and its involvement on the disturbances of muscle tonus and strength perception, as well its connection with others systems involved on the motor control. Methods: The bibliographic revision was realized on period between 1964 until nowadays. The study was based on experimental and review articles beyond of texts-books chapters. Results: The GTO is innervated by lb afferent (af) fiber which is always activated when there are tension in the muscle, inhibiting, by means of $\mathrm{lb}$ inhibitory interneuron (IN), the motor neuron which is generating the contraction. Moreover, an Ib excitatory IN facilitates the antagonist motor neurons by means of two synapses. The stimulus that arrives to lb IN comes from of many origins, including many peripherical af and excitatory and inhibitory descendant stimulus of superior centers. Conclusion: The GTO acts together with the spindle muscle on regulation of complacency and tonus muscle; its involved on proprioception mediation and has an important function on the normal locomotor rhythm.

\section{Keywords: Mechanoreceptors, Proprioception, Muscle tonus.}

Citation: Taneda M, Pompeu JE. Physiology and importance of Golgi tendon organ for the normal motor control. Rev Neurocienc 2006; 14(1):037-042.

Trabalho realizado: Universidade Paulista Campus Tatuapé.

1 - Graduação em Fisioterapia pela Universidade Paulista - UNIP

2 - Fisioterapeuta e Mestre em Neurociências e Comportamento pela Universidade de São Paulo - USP. Professor adjunto e coordenador do curso de Fisioterapia da UNIP.

Endereço para correspondência: Rua Constantino Burato, 55. Vila Barros, Guarulhos. São Paulo. CEP: 07192-040. Brasil. Tel: 6404-5215. E-mail: marcotaneda@ig.com.br, jep72@terra.com.br 


\section{INTRODUÇÃO}

Os órgãos tendinosos de Golgi (OTG) são receptores sensoriais encapsulados delgados, com 1 milímetro de comprimento e 0,1 milímetro de diâmetro. Estão localizados na junção miotendínea (entre o músculo e seu tendão), onde fibras colágenas (originadas no tendão) se prendem às extremidades de grupos de fibras extrafusais. No interior da cápsula do OTG, os feixes colágenos se dividem em fascículos finos que formam uma estrutura trançada 1 . O OTG está conectado em série com as fibras musculares paralelas ao tendão?

O OTG é inervado por axônio aferente (af) lb, que perde sua mielinização após entrar na cápsula, ramificando-se em muitas terminações delgadas, que se enroscam, por sua vez, por entre os fascículos trançados do colágeno. O estiramento do OTG causa o estiramento dos feixes de colágeno. Isso resulta na compressão das terminações sensoriais, fazendo com que fiquem ativadas. Estiramentos muito pequenos do OTG podem deformar as terminações nervosas que cursam por entre as fibras de colágeno ${ }^{(1)}$. Portanto, as fibras lb disparam sempre que há tensão no músculo, seja por uma contração ou por um alongamento. Porém, o alongamento passivo do músculo não é tão efetivo na ativação dos $\mathrm{OTG}^{3-5}$

Stephens et al. ${ }^{4}$ analisaram as respostas de 13 OTG a fim de graduar a força de 29 unidades motoras no gastrocnêmio medial de gatos em 5 experimentos, e constataram que a atividade total aferente lb durante o estiramento passivo muscular é muito menor que durante a atividade de contração muscular.

No OTG as fibras colágenas no tendão são mais rígidas que as fibras musculares com que estão em série. Por conseguinte, a maior parte do estiramento acontece nas fibras musculares, mais complacentes, ocorrendo uma deformação mecânica muito menor no OTG. Quando o músculo se contrai, as próprias fibras musculares tracionam diretamente as fibras colágenas, transmitindo, com maior eficiência, o estiramento para o OTG. Como resultado, os OTG sempre respondem de forma mais intensa à contração muscular do que ao seu estiramento ${ }^{1}$.

O OTG possui uma resposta dinâmica e uma resposta estática. A resposta dinâmica surge quando a tensão muscular aumenta subitamente e o OTG, por conseqüência, responde de forma intensa. A resposta estática ocorre em uma fração de segundo após a resposta do OTG, sendo que este volta a um nível inferior de descarga no estado estável, que é quase diretamente proporcional à tensão muscular. Assim, o OTG proporciona ao sistema nervoso informações sobre o grau de tensão em cada pequeno segmento do músculo ${ }^{6,7}$.

A terminação af Ib influencia o músculo homônimo através de um interneurônio (IN) inibitório lb que inibe o neurônio motor que está gerando a força. Esse processo é denominado inibição autogênica (ou autógena) que produz um mecanismo de retroalimentação negativa impedindo o desenvolvimento de tensão demasiada no músculo. Além disso, os neurônios motores antagonistas são facilitados através de duas sinapses por um IN excitatório lb. Esse processo é a excitação recíproca ${ }^{5}$. A via lb pode ser dissináptica ou trissináptica ${ }^{8-10}$.

O potencial pós-sináptico inibitório é esperado, geralmente, para os sinergistas, porém há poucos casos em que isso ocorre nos antagonistas ${ }^{8,10}$. Watt et $a l .^{8}$ examinaram af $\mathrm{lb}$ do gastrocnêmio medial de gatos e constataram que o af lb ocasionava potencial pós-sináptico excitatório em 18\% dos motoneurônios sinergistas e em $28 \%$ dos antagonistas, e potencial pós-sináptico inibitório em $41 \%$ dos sinergistas e em $19 \%$ dos antagonistas.

Esse arranjo reflexo (inibição do músculo homônimo e seus sinergistas) sugere um tipo de função protetora do OTG, pois ele pode manter a freqüência de disparo do neurônio motor em um nível seguro evitando as microrrupturas espontâneas dos tecidos músculo-tendinosos. O IN lb exerce a maior influência sobre os neurônios motores associados às unidades motoras rápidas glicolíticas, pois elas são as maiores produtoras de força entre os três tipos de unidades motoras. O IN Ib tem a menor influência sobre os neurônios motores das unidades motoras lentas, pois estas produzem os menores níveis de força ${ }^{5}$. O OTG e o fuso muscular exercem influências opostas. Por essa razão, alguns pesquisadores se referem ao reflexo do OTG como reflexo miotático inverso ou reflexo de estiramento inverso ${ }^{5}$.

O objetivo do presente estudo é, portanto, relatar a fisiologia do OTG e seu envolvimento em distúrbios do tônus muscular e na percepção da força, bem como sua interação com outros sistemas envolvidos no controle motor normal.

\section{MÉTODO}

A revisão da literatura foi realizada utilizando as bases de dados do Medline, Web of Science e Lilacs, no período de 1964 até os dias atuais, utilizando os descritores: spinal cord, motoneurones, Ib afferents, Golgi tendon organ, autogenic inhibition, Ib inhibition e Ib interneurones.

O critério de seleção de palavras chaves foi a utilização dos descritores citados nos artigos clássicos sobre o tema de estudo. Após a busca com os descritores clássicos, efetuou-se uma nova pesquisa utilizando-se dos demais temas sugeridos pelos autores selecionados. Por se tratar de um tema relacionado à neurofisiologia, sendo esta considerada como área básica, os descritores da saúde não abrangem tais estudos. Por este motivo, houve a necessidade de se adotar o procedimento descrito acima. 
O critério de inserção utilizado no presente estudo foi a seleção de artigos cujo tema relacionava-se diretamente com o assunto abordado. Foram inseridos artigos experimentais em humanos e animais, além de artigos de revisão e capítulos de livros-textos.

\section{RESULTADOS}

\section{Função do OTG e vias do IN Ib:}

Funcionalmente, o OTG possui mais que um papel protetor. Por causa de sua excelente sensibilidade à força muscular e de sua localização anatômica, o OTG atua juntamente com o fuso muscular a fim de regular o tônus e a complacência musculares ${ }^{5}$.

O fuso muscular e o OTG possuem um papel importante na mediação da propriocepção subconsciente ${ }^{6}$. O sinal desses dois receptores é total ou quase que totalmente para o propósito do controle muscular intrínseco. Eles transmitem uma enorme quantidade de informações, não somente para a medula espinal como também para o cerebelo e para o córtex cerebral, ajudando cada uma dessas partes do sistema nervoso em sua função de controlar a contração muscular? .

Uma provável função do reflexo tendinoso de Golgi é a equalização das forças contráteis de fibras musculares distintas, ou seja, as fibras que exercem tensão excessiva são inibidas pelo reflexo, enquanto as que exercem muito pouca tensão são mais excitadas, devido à ausência da inibição reflexa. Isso dispersaria a carga muscular por todas as fibras e impediria os danos a áreas isoladas do músculo, onde pequeno número de fibras poderia ser sobrecarregado ${ }^{7}$.

.A influência do OTG sobre um grupo neuronal motor alfa pode ser regulada centralmente por estímulos convergentes ao IN Ib. A influência do IN lb sobre a excitabilidade dos neurônios motores depende das entradas combinadas de muitas fontes, tanto centrais como periféricas ${ }^{1,6}$. Os estímulos convergem ao IN Ib das mais variadas fontes, incluindo os af $\mathrm{lb}$, af la, af contralaterais la e lb, af II, terminações af da cápsula articular, af cutâneos, af do reflexo flexor e estímulos excitatórios e inibitórios descendentes dos centros superiores s,5,10-13. $^{1}$.

Harrison e Jankowska ${ }^{12}$ pesquisaram as vias para o IN Ib a fim de quantificá-las através de fórmulas matemáticas e gráficos. Porém, não puderam chegar a um resultado específico devido à existência de muitas variáveis.

Para o controle motor, a importância funcional dessa convergência pode ser considerada no contexto do controle fino da força de preensão durante a manipulação física de objetos. Por exemplo, ao segurar-se um livro entre o dedo indicador e o polegar, contrapondo-se o peso com a força dos dedos. Se essa força, por acaso for um pouco baixa, o objeto pode começar a deslizar entre os dedos, e os receptores cutâneos serão então excitados, e com o estímulo inibitório dos aferentes cutâneos ao IN Ib o efeito inibitório do OTG pode ser reduzido, permitindo um aumento da força de preensão que interrompa esse deslizamento ${ }^{5}$.

Outro exemplo da função do OTG seria quando a mão entra em contato pela primeira vez com um objeto, a força muscular é intensamente inibida pela ativação combinada dos OTG e de aferentes cutâneos, permitindo, dessa forma, a redução imediata da força muscular a fim de amaciar o tato. Vias descendentes podem regular a intensidade desse efeito inibitório, aumentando-o, se o objeto for frágil, ou diminuindo a inibição, caso seja desejado um contato mais forte ${ }^{1}$.

Podemos ainda exemplificar a função do OTG quando o membro inferior encontra um obstáculo durante a caminhada, os aferentes cutâneos disparam ajudando a diminuir a força muscular a fim de não forçar o membro contra o obstáculo (a facilitação dos músculos antagonistas pode contribuir para frear o movimento) ${ }^{10}$.

Harrison e Jankowska (1985a) citados por Malmgren ${ }^{10}$ demonstraram que os aferentes do reflexo flexor efetivamente excitam o IN Ib. Em relação aos af capsulares, propõe-se que eles possam influenciar o OTG da mesma forma que os af cutâneos o fazem ${ }^{10}$.

Os tratos descendentes que excitam o IN Ib são o corticoespinal e o rubroespinal, enquanto os que o inibem são o reticuloespinal dorsal (reticuloespinal bulbar dopaminérgico) e o reticuloespinal noradrenérgico 9,10,13,14.

A força e o sinal da transmissão sináptica (no caminho do reflexo espinal) pode ser alterado durante as tarefas motoras. As fibras af $\mathrm{lb}$ vindas dos músculos extensores possuem um efeito inibitório no neurônio motor extensor na ausência da atividade locomotora, porém, durante a locomoção, a mesma fibra lb produz um efeito excitatório no neurônio motor extensor, e deprime a transmissão em caminho bissináptico lb. Esse fenômeno é referido como state-dependent reflex reversa/15,16.

\section{Projeções Corticais e Cerebelares do OTG:}

As informações sobre uma modalidade sensorial específica, como a força ou o comprimento musculares, e sobre uma região específica do corpo são separadas em cada nível do sistema da coluna dorsal-lemnisco medial e processadas por neurônios que formam grupos funcionais separados. A informação sensorial atinge o córtex somatossensorial que é organizado somatotopicamente. Há quatro regiões distintas: áreas 1, 2, 3a e 3b (de acordo com as subdivisões do córtex feitas por Brodmann). Grande parte das fibras talâmicas termina na área 3, e então projeta-se para as áreas 1 e 2. Estímulos dos receptores de estiramento, dos OTG, e de receptores articulares vão para a área 3a. Informações dos receptores cutâneos vão para a área 3b. Estímulos dos receptores musculares e articulares podem se 
sobrepor na área 3a, porém, ocorre uma segmentação nas projeções subseqüentes para as áreas 2 e 1. A partir disso, a informação proprioceptiva é transmitida para a área 5 do córtex parietal. Todas essas projeções provêm do lado contralateral do corpo ${ }^{17}$.

Das vias aferentes para o cerebelo, os tratos mais importantes são os tratos espinocerebelares (dorsais e ventrais). Os sinais dos tratos espinocerebelares dorsais são provenientes, principalmente, dos fusos musculares e, em menor escala, de outros receptores somáticos em todo o corpo, como OTG, grandes receptores táteis na pele e receptores articulares. Por outro lado, os tratos espinocerebelares ventrais recebem menos informações dos receptores periféricos. Em vez disso, eles são excitados, principalmente, pelos sinais motores que chegam dos cornos anteriores da medula espinal a partir: do cérebro (pelos tratos corticoespinal e rubroespinal); e dos geradores de padrão internos da própria medula espinal. Assim, essa via de fibras ventrais tem o papel de informar ao cerebelo que sinais motores chegaram aos cornos anteriores; essa retroalimentação é denominada cópia eferente da pulsão motora dos cornos anteriores ${ }^{18}$.

As vias espinocerebelares podem transmitir impulsos com velocidade de até $120 \mathrm{~m} / \mathrm{s}$, que é a condução mais rápida de qualquer via do sistema nervoso central, e é importante para o conhecimento instantâneo, pelo cerebelo, das alterações nas ações musculares periféricas ${ }^{18}$.

\section{OTG na Percepção da Força:}

Há pelo menos duas fontes para a percepção da força gerada pelo músculo. Primeira, através dos OTG. Segunda, a percepção da força pode derivar de um correlato neural interno ou de uma cópia do comando motor (descarga conseqüente) enviada ao conjunto de neurônios motores. Muito provavelmente, esse sinal é enviado aos centros sensoriais do cérebro e pode refletir a magnitude do sinal motor descendente. Essas sensações mediadas centralmente são chamadas percepção do esforço, enquanto as sensações originadas perifericamente são chamadas de percepção da força ou tensão ${ }^{17}$.

Durante a fadiga tanto a força quanto o disparo do lb diminuem, e não há indícios de que o acúmulo de metabólitos possa causar um efeito excitatório nesses aferentes ${ }^{(4)}$. Porém, na ausência de força ou de fadiga muscular, a percepção da força e do esforço fornecem informações iguais, ou seja, à medida que aumenta o comando motor, aumenta também a taxa de disparo dos OTG sinalizando a força da contração. É impossível diferir, nessas condições, qual das duas fontes de informações sobre a força é utilizada ${ }^{17}$.

Alguns experimentos indicam que, quando aumentase o comando motor enviado a um músculo, aumentase proporcionalmente a magnitude percebida da força de contração, mesmo quando a força exercida pelo músculo permanece constante. Neste caso, como a força é mantida, aumenta-se o impulso neural necessário para gerar a força quando ocorre a fadiga muscular, e a amplitude percebida da força aumenta. Parece pouco provável que a superestimação da força seja baseada em descargas dos OTG, pois elas permanecem constantes ou diminuem (se houver adaptação) enquanto o músculo continua a gerar uma força constante ${ }^{17}$.

Durante outros estados e condições (exemplo: fraqueza ou paresia) que afetam a capacidade de geração de força do músculo também são aparentes as alterações da força e do peso ${ }^{17}$.

\section{Envolvimento do OTG em Distúrbios do Tônus Muscular:}

Hipertonia caracteriza basicamente dois estados anormais do tônus muscular: a espasticidade e a rigidez ${ }^{5}$. Tanto na espasticidade quanto na rigidez a inibição Ib pode estar diminuída ou até ausente ${ }^{9,13}$

A espasticidade normalmente é acompanhada pela resistência em canivete e os reflexos de contração são exagerados, ou seja, ocorre um aumento da responsividade dos neurônios motores alfa ao estímulo sensorial do tipo la. O fenômeno do canivete refere-se à resistência maior à manipulação passiva durante a faixa inicial de amplitude de movimento (seja a flexão ou a extensão) e ao rápido declínio da resistência quando a amplitude da movimentação do membro é aumentada. A resistência da resposta inicial em canivete decorre da hiperatividade do reflexo de estiramento, enquanto - OTG provavelmente está envolvido no desencadeamento súbito do reflexo de estiramento ${ }^{5}$.

Rymer et al..$^{19}$ propuseram que o sinal do canivete possa ser mediado por efeitos inibitórios centrais dos grupos aferentes III e IV tendo a clássica ação do reflexo flexor. Esses grupos aferentes respondem a estímulos térmico, nocivo, químico, e mecânico. Os axônios do grupo I são fibras largamente mielinizadas com velocidade de condução de 72 a 120 m/s, e estão relacionados com os OTG e a terminações primárias do fuso muscular. Os axônios do grupo II são fibras pouco mielinizadas com velocidade de condução de 24 a 72 m/s, e estão relacionados com a terminações secundárias do fuso muscular. Os axônios do grupo III são menos mielinizadas do que o grupo Il e possuem velocidade de condução de $24 \mathrm{~m} / \mathrm{s}$, enquanto os axônios do grupo IV são fibras amielínicas com velocidade de condução de até $2,5 \mathrm{~m} / \mathrm{s}^{20}$.

De acordo com Rymer et al..$^{19}$, as propriedades de baixa sensitividade dos receptores de estiramento podem fornecer alguma espécie de função protetora parecida com a função do OTG. Isso complementa as informações obtidas no presente estudo (onde é relatado que o IN Ib recebe estímulos das mais variadas fontes) 
Delwaide e Oliver ${ }^{9}$ e Rymer et al. ${ }^{19}$ afirmaram que a via do OTG não é responsável pelo mecanismo do efeito canivete. Segundo Rymer et al. ${ }^{19}$, a via do OTG não combina com o modelo do efeito canivete, pois nesta inibição a força muscular está longe de diminuir, enquanto a atividade do OTG declina juntamente com a redução da tensão muscular.

O efeito canivete pode ocorrer devido aos receptores de terminações nervosas livres musculares para funcionar primariamente como um reflexo de retirada ${ }^{21}$.

A inibição recíproca la, inibição lb e inibição pré-sináptica também podem contribuir para os reflexos de estiramentos exagerados, em particular, a manifestação do clônus ${ }^{22}$.

O funcionamento do IN Ib na rigidez de Parkinson tem sido muito estudado, pois a eficácia desse IN é reduzida nessa doença e na espasticidade ${ }^{9,13,14}$. Para explicar a perda da atividade do IN lb e maior atividade do IN inibitório la, Delwaide et al. ${ }^{13}$ e Fine et al. ${ }^{14}$ atribuíram esses efeitos ao trato reticuloespinal, pois este exerce (em animais) efeitos opostos nesses dois IN inibitórios. Delwaide et al. ${ }^{13}$ propuseram que os núcleos gigante-celulares, que recebem projeções da substância negra ${ }^{23}$, podem estar mais ativados causando modificações nos IN. A partir disso, podese traçar uma seqüência de disfunções começandose pela substância negra, passando pelos núcleos reticulares e chegando aos IN que causam a rigidez clínica característica.

Potter et al. ${ }^{24}$ demonstraram que a estimulação de alta freqüência dos núcleos subtalâmicos é capaz de restaurar a reduzida inibição autogênica em pacientes com doença de Parkinson.

\section{Estudos do OTG na Locomoção:}

A locomoção não é baseada no encadeamento de reflexos, uma vez que a desaferentação não é capaz de abolir o programa motor no caminhar de gatos espinalizados ${ }^{25}$. Porém, esses aferentes musculares podem funcionar como reguladores do ato motor do caminhar, adaptando o programa locomotor central de acordo com as necessidades do meio 25,26 .

Através de uma estimulação fraca do nervo plantar no maléolo interno da articulação do tornozelo, Shoji et al. ${ }^{27}$ demonstraram que a inibição proveniente da via lb vinda do nervo do gatrocnêmio medial produziu uma inibição de curta latência da atividade eletromiográfica do sóleo em humanos. Os autores também demonstraram que menor depressão eletromiográfica ocorria após a estimulação do nervo plantar durante a fase de apoio da locomoção comparado com a flexão plantar tônica ou dinâmica. Em adição, os OTG, por fornecerem uma retroalimentação da força do músculo homônimo, possuem uma contribuição substancial para a atividade extensora do tornozelo durante a locomoção de gatos descerebrados ${ }^{28}$.

Duysens e Pearson ${ }^{25}$, em suas pesquisas em gatos, notaram que quando o tríceps sural está em contração a atividade dos músculos flexores é inibida. Eles propuseram que, quando os músculos extensores estão se contraindo, os OTG desses músculos disparam e inibem a geração da flexão. Este fenômeno pode ser importante durante o ato normal de caminhar, porque os músculos extensores na fase de apoio precisam suportar o peso do corpo e, ao inibirem (via OTG) a geração da flexão, estariam evitando uma "desincronia" durante a locomoção que seria totalmente prejudicial no referido ato. Os autores sugerem que o início da atividade flexora durante o caminhar depende da cessação da contração dos músculos extensores da perna, ou seja, os aferentes extensores do grupo lb precisam cessar o seu disparo. Isto levou os autores à conclusão de que os OTG dos músculos extensores são importantes mediadores da passagem da fase de apoio para a fase de balanço durante a locomoção.

Em pesquisas com gatos espinalizados, Conway et al. ${ }^{26}$, constataram que a estimulação dos aferentes do grupo I pode reiniciar a atividade rítmica: um aumento da atividade dos aferentes lb do OTG aboliu o ritmo locomotor e levou à uma atividade extensora tônica do membro ipsilateral, enquanto o ritmo do membro contralateral permaneceu inalterado. A capacidade de reiniciar o ritmo locomotor parece vir somente de aferentes extensores do grupo I e em particular dos aferentes extensores do grupo lb, pois a vibração visando a ativação das fibras la não obteve alterações no ritmo indicando que esses aferentes não influenciavam o ritmo. Isto tudo levou os autores a concluírem que a contração dos músculos extensores provê estímulos adequados (via OTG) para reiniciar o ritmo locomotor.

Lundberg et al. (1977) citados por Pierrot-Deseilligny et al. ${ }^{11}$, em estudos em gatos espinais, demonstraram que uma estimulação de aferentes cutâneos de baixo limiar provenientes do membro ipsilateral facilita a transmissão sináptica dos aferentes lb para os motoneurônios. Porém, Pierrot-Deseilligny et al. ${ }^{11}$ mostraram que, em humanos normais, uma estimulação cutânea tátil do pé fortemente deprime a via reflexa do lb para os motoneurônios favorecendo a contração muscular no joelho (quadríceps e cabeça curta do bíceps femoral). Este fenômeno é observado somente quando aferentes cutâneos do pé são estimulados. Notaram, também, que essa depressão só ocorre quando a sensação cutânea é percebida na área da pele (sola do pé, pontas dos dedos) em contato com o chão durante a caminhada. Segundo os autores, este mecanismo pode ser um dos contribuintes para a locomoção humana. 
Além dos estudos citados anteriormente ${ }^{25,26,29}$, vários outros estudos posteriores foram feitos ${ }^{15,16,30-34}$ e as conclusões às quais os autores chegaram (seja direta ou indiretamente) podem ser resumidas da seguinte forma: (A). A estimulação elétrica dos aferentes do grupo I pode inibir a geração da flexão durante o ato locomotor; (B). Aferentes extensores do grupo lb podem excitar os motoneurônios extensores durante a locomoção; e (C). O ritmo locomotor pode ser reiniciado pelos aferentes extensores lb.

\section{CONCLUSÕES}

O OTG atua juntamente com o fuso muscular na regulação do tônus e da complacência musculares; está envolvido na mediação da propriocepção e possui um papel importante no ritmo locomotor normal. Atua através de uma complexa integração entre diversos aferentes oriundos de receptores periféricos e também está sob influência suprassegmentar, sendo que o padrão final do comportamento motor é o resultado desta interação.

\section{REFERÊNCIAS BIBLIOGRÁFICAS}

1. Guez C, Gordon J. Os Músculos e os Receptores Musculares. Reflexos Espinhais. In: Kandel ER, Schwartz JH, Jessel TM. Fundamentos da Neurociência e do Comportamento. Rio de Janeiro: Guanabara Koogan; 2000; p.403-422

2. Houk JC, Henneman E. Responses of Golgi tendon organs to active contractions of the soleus muscle of the cat. J Neurophysiol 1967; 30: 466-481.

3. Jansen JKS, Rudjord T. On the silent period and Golgi tendon organs of the soleus muscle of the cat. Acta Physiol Scand 1964; 62: 364-379.

4. Stephens JA, Reinking RM, Stuart DG. Tendon organs of cat medial gastrocnemius: responses to active and passive forces as a function of muscle length. J Neurophsiol 1975; 38: 1217-1231.

5. Iyer MB, Mitz AR, Winstein C. Sistemas Motores. In: Cohen H. Neurociência para Fisioterapeutas. São Paulo: Manole; 2001; p.221, 230, 231 e 233.

6. Umphred DA, McCormack GL. Classificação das Técnicas de Tratamento Facilitadoras e Inibidoras Mais Utilizadas. In: Umphred DA. Fisioterapia Neurológica. São Paulo: Manole; 1994; p.116-117.

7. Guyton AC, Hall JE. Funções Motoras da Medula Espinhal. Os reflexos Medulares. In: Guyton AC, Hall JE. Tratado de Fisiologia Medica. Rio de Janeiro: Guanabara Koogan; 2002; p.582, 586.

8. Watt DGD, Stauffer EK, Taylor A, Reinking RM, Stuart DG. Analysis of muscle receptor connections by spike-triggered averaging.1. Spindle primary and tendon organ afferents. J Neurophysiol 1976; 39: 1375-1392.

9. Delwaide PJ, Oliver E. Short-latency autogenic inhibition (lb inhibition) in human spasticity. J Neurol Neurosurg Psychiatr 1988; 51: 1546-1550.

10. Malmgren K. On premotoneuronal integration in cat and man. Acta Physiol Scand Suppl 1988; 576: 1-53.

11. Pierrot-Deseilligny E, Bergego C, Katz R, Morin C. Cutaneous depression of Ib reflex pathways to motoneurones in man. Exp Brain Res 1981; 42 351-361.

12. Harrison PJ, Jankowska E. Organization of input to the interneurones mediating group I non-reciprocal inhibition of motoneurones in the cat. J Physiol 1985; 361: 403-418.

13. Delwaide PJ, Pepin JL, Maertens de Noordhout A. Short-latency autogenic inhibition in patients with parkinsonian rigidity. Ann Neurol 1991; 30: 83-89

14. Fine EJ, Hallett M, Litvan I, Tresser N, Katz D. Dysfunction of Ib (autogenic) spinal inhibition in patients with progressive supranuclear palsy. Mov Disord 1998; 13:668-672

15. Pearson K, Gordon J. Spinal Reflexes. In: Kandel ER, Schwartz JH, Jessel TM. Principles of Neural Science. New York: McGraw-Hill; 2000; p.724.

16. Cook AS, Woollacott MH. Control of Normal Mobility. In: Cook AS, Woollacott MH. Motor Control. Theory and Practical Applications. Philadelphia Lippincott Williams and Wilkins; 2001; p.320 e 321

17. Jones LA. Sentidos Somáticos 3: Propriocepção. In: Cohen H. Neurociência para Fisioterapeutas. São Paulo: Manole; 2001; p.116, 117, 123 e 124.
18. Guyton AC, Hall JE. O Cerebelo, os Gânglios da Base e o Controle Motor Global. In: Guyton AC, Hall JE. Tratado de Fisiologia Medica. Rio de Janeiro: Guanabara Koogan; 2002; p.605

19. Rymer WZ, Houk JC, Crago PE. Mechanisms of clasp-knife reflex in an animal model. Exp Brain Res 1979; 37: 93-113.

20. Abrahams VC. Group III and IV receptors of skeletal muscle. Can J Physiol Pharmacol 1986; 64(4): 509-514

21. Cleland CL, Rymer WZ. Functional properties of spinal interneurons activated by muscular free nerve endings and their potential contributions to the clasp-knife reflex. J Neurophysiol 1993; 69(4): 1181-1191.

22. Masakado Y, Kagamihara Y, Takahashi O, Akaboshi K, Muraoka Y, Ushiba J. Post-activation depression of the soleus H-reflex in stroke patients. Electromyogr Clin Neurophysiol 2005; 45(2): 115-22.

23. Chronister RB, Walding JS, Aldes LD, Marco LA. Interconnections between substantia nigra reticulata and medullary reticular formation. Brain Res Bul 1988; 21: 313-317

24. Potter M, Illert M, Wenzelburger R, Deuschl G, Volkmann J. The effect of subthalamic nucleus stimulation on autogenic inhibition in Parkinson disease. Neurology 2004; 63(7): 1234-9.

25. Duysens J, Pearson KG. Inhibition of flexor burst generation by loading ankle extensor muscles in walking cats. Brain Res 1980; 187: 321-332.

26. Conway BA, Hultborn $\mathrm{H}$, Kiehn O. Proprioceptive input resets central locomotor rhythm in the spinal cat. Exp Brain Res 1987; 68: 643-656.

27. Shoji J, Kobayashi K, Ushiba J, Kagamihara Y, Masakado Y. Inhibition from the plantar nerve to soleus muscle during the stance phase of walking. Brain Res 2005; 1048: 48-58

28. Donelan JM, Pearson KG. Contribution of force feedback to ankle extensor activity in decerebrate walking cats. J Neurophysiol 2004; 92: 2093-2104.

29. Pearson KG, Ramirez JM, Jiang W. Entrainment of the locomotor rhythm by group $\mathrm{lb}$ afferents from ankle extensor muscles in spinal cats. Exp Brain Res 1992; 90: 557-566

30. Pearson KG, Collins DF. Reversal of the influence of group lb afferents from plantaris on activity in medial gastrocnemius muscle during locomotor activity. J Neurophysiol 1993; 70: 1009-1017

31. Gossard JP, Brownstone RM, Barajon I, Hultborn H. Transmission in a locomotor-related group lb pathway from hindlimb extensor muscles in the cat. Exp Brain Res 1994; 98: 213-228

32. Pearson KG. Proprioceptive regulation of locomotion. Curr Opin Neurobio 1995; 5: 786-791.

33. Whelan PJ, Hiebert GW, Pearson KG. Stimulation of the group I extensor afferents prolongs the stance phase in walking cats. Exp Brain Res 1995; 103: 20-30.

34. Stephens MJ, Yang JF. Short latency, non-reciprocal group I inhibition is reduced during the stance phase of walking in humans. Brain Res 1996; 743: 24-31. 